\title{
Case of Langerhans Cell Histiocytosis That Mimics Meningioma in CT and MRI
}

\author{
Ming Zhu, M.D., Ph.D., ${ }^{1}$ Bing-Bing Yu, M.D., Ph.D., ${ }^{1}$ Ji-Liang Zhai, M.D., Ph.D., ${ }^{2}$ Gang Sun, M.D., Ph.D. ${ }^{1}$ \\ Department of Medical Imaging, ${ }^{1}$ Jinan Military General Hospital, Jinan, China \\ Department of Radiology, ${ }^{2}$ The Branch of Taian Central Hospital, Taian, China
}

Langerhans cell histiocytosis $(\mathrm{LCH})$ is a rare disorder histologically characterized by the proliferation of Langerhans cells. Here we present the case of a 13-year-old girl with LCH wherein CT and MRI results led us to an initially incorrect diagnosis of meningioma. The diagnosis was corrected to LCH based on pathology findings. An intracranial mass was found mainly in the dura mater, with thickening of the surrounding dura. It appeared to be growing downward from the calvaria, pressing on underlying brain tissue, and had infiltrated the inner skull, causing a bone defect. The lesion was calcified with the typical dural tail sign. The dural origin of the lesion was verified upon surgical dissection. There are no previous reports in the literature describing LCH of dural origin presenting in young patients with typical dural tail signs and meningioma-like imaging findings. The current case report underscores the need for thorough histological and immunocytochemical examinations in LCH differential diagnosis.

Key Words : CT $\cdot$ MRI $\cdot$ Meningioma $\cdot$ Langerhans cell histiocytosis.

\section{INTRODUCTION}

Langerhans cell histiocytosis (LCH) is a rare and benign chronic inflammatory disease of unknown etiology characterized by the idiopathic proliferation of monoclonal histiocytes. It has variable clinical features and occurs most frequently in children ${ }^{4)}$. LCH presents as three main clinical subtypes : unifocal (eosinophilic granuloma) involving a single organ; multifocal (a.k.a. Hand-Schuller-Christian disease) presenting as a disseminated disease; and systemic (Abt-Letterer-Siwe disease) ${ }^{6}$. Of the three subtypes, unifocal LCH is the most common presentation and bone is the most frequently affected tissue ${ }^{8)}$. The progression of the condition varies greatly, ranging from spontaneous regression to rapid progression, with or without recurrence and long-lasting sequelae $e^{2)}$.

The diagnosis of LCH is based on characteristic histopathological features. Under light microscopy, tumor cells present as large histiocytes with grooved nuclei, and mononuclear histiocytes and multinucleated giant cells are intermixed with eosinophils, lymphocytes, plasma cells, and neutrophil polymorphs ${ }^{8)}$. Immunohistochemistry shows S-100 protein and CD1a expression in Langerhans cells ${ }^{6}$. Electron microscopy may also reveal "tennis racket" shaped cytoplasmic inclusions within histiocytes called Birbeck granules, which is the gold standard for LCH diagnosis. Imaging often shows a round or elliptical transparent bone defect. The edge of the defect may be clear or unclear, and the defect can involve inner and outer areas of cranial bones ${ }^{1)}$. Cranial and intracranial changes measured by MRI include : 1) lesions of the craniofacial bone and skull base with or without soft-tissue extension; 2) intracranial extra-axial changes (hypothalamic-pituitary region, meninges, circumventricular organs); 3 ) intracranial intra-axial changes (white matter and gray matter); and 4) cerebral atrophy ${ }^{9}$.

\section{CASE REPORT}

A 13-year-old female patient sought treatment complaining of chronic headaches and dizziness that she had been experiencing for the preceding year. Head CT results showed a high density mass on the right calvaria with calcification spots and an adjacent skull defect with uneven borders (Fig. 1A, B). Noncontrast MRI revealed a heterogeneous area of signal intensity in T1WI and a high-intensity mass with low-intensity shadows within it in T2WI. Contrast MRI showed an intensified mass of $3.5 \times 3.8 \times 2.0 \mathrm{~cm}$ with a heterogeneous signal. The lesion was connected with the dura mater through the wide base, showing

- Received : September 25, 2014 • Revised : January 23, 2015 •Accepted : January 26, 2015

- Address for reprints : Gang Sun, M.D., Ph.D.

Department of Medical Imaging, Jinan Military General Hospital, 25 Shifan Road, Jinan 250031, China

Tel : +86-531-51666864, Fax : +86-531-51666486, E-mail : cjr.sungang@vip.163.com

- This is an Open Access article distributed under the terms of the Creative Commons Attribution Non-Commercial License (http://creativecommons.org/licenses/by-nc/3.0) which permits unrestricted non-commercial use, distribution, and reproduction in any medium, provided the original work is properly cited. 
a dural tail sign and pressing downward on adjacent brain tissue. A strip-shape intensified signal was observed in the adjacent skull (Fig. 1C, D). Low-signal areas in the T2WI nd areas without intensification demonstrated by contrast MRI were identical to calcified areas in CT result.

The lesion was diagnosed initially as a meningioma and the patient underwent a craniotomy. During the surgery, it was revealed that the lesion originated in the dura mater and had
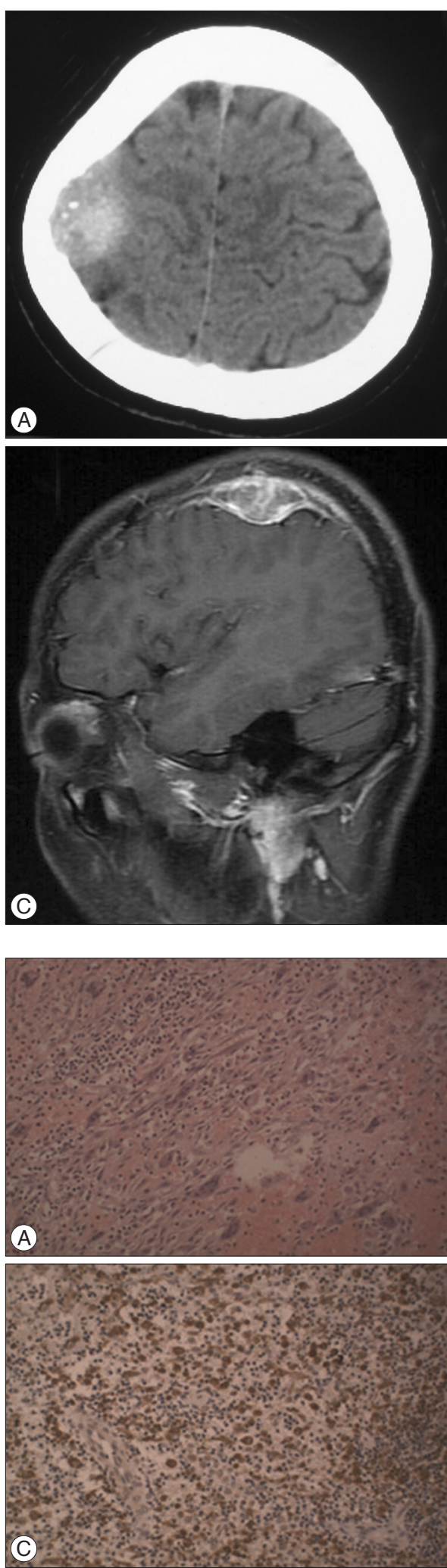

B
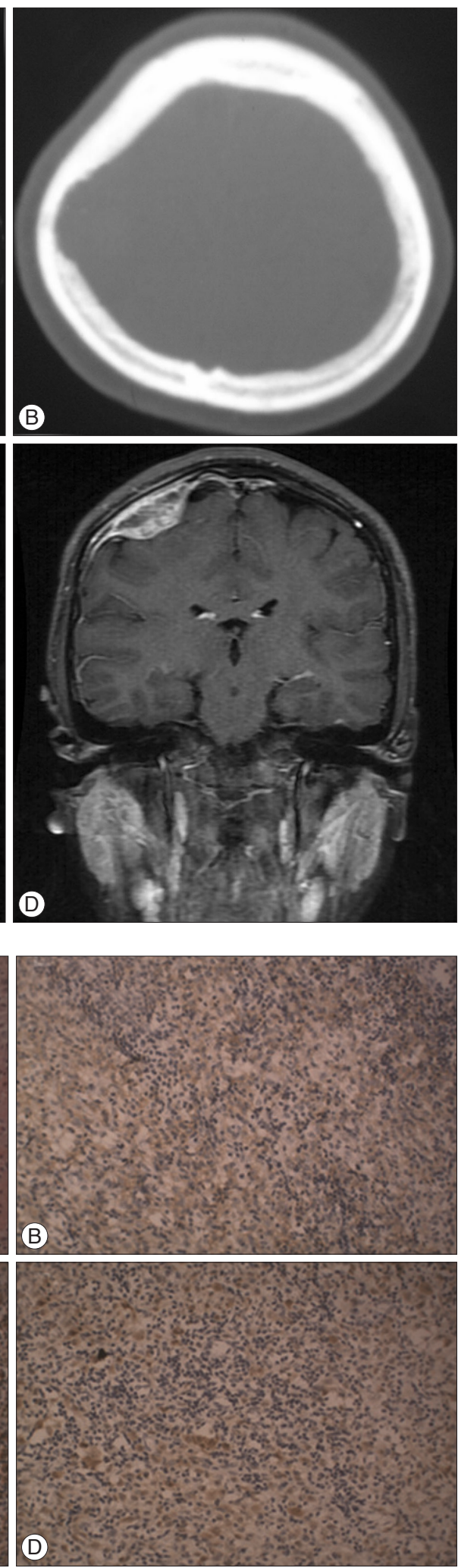

Fig. 1. A : Plain CT scan. Soft-tissue window, high-density mass on the right calvaria with internal calcification spots. B : Plain CT scan. Bone window and adjacent bone deficit with unclear edges. $C$ and $D$ : Enhanced MRI revealing an irregularly shaped tumor with clear boundaries and a wide base attached to the meninges (sagittal and coronal planes, respectively).

Fig. 2. Histopathological demonstration of $\mathrm{LCH}$. Hematoxylin-eosin staining of the resected mass revealed a large number of Langerhans cells, multinucleated giant cells, and diffuse eosinophil and lymphocyte infiltration (A). Immunohistochemistry revealed the presence of membranous CD1a (B), cytoplasmic CD68 (C), and nuclear S-100 (D). Original magnification : $\times 200$. 
grown downward, pressing on the brain tissue and invading the inner skull. The dura around the tumor was thickened. The tumor was elastic and had a rich blood supply, indicating endogenous angiogenesis. The tumor and surrounding dura were removed, and artificial dura material was used to fill the resultant defect in the dura mater. Histopathological examination revealed a grey-white irregular tissue with a dark brown crosssection. Microscopy revealed a large number of proliferated Langerhans cells and multinuclear giant cells, as well as infiltration of eosinophils and lymphcytes (Fig. 2). Immunohistochemical examination indicated that the tumor tissue was CD1a-, CD68-, and S-100-positive. Therefore, a final diagnosis of LCH was confirmed.

\section{DISCUSSION}

Here we reported the case of a 13-year-old female patient with a unifocal LCH of dural origin that was initially misdiagnosed as a meningioma based on imaging findings, but was later determined by the pathological examination to be LCH. To our knowledge, there have been no prior reports of teenagers having duraoriginating calvarial LCHs that exhibit the typical dural tail sign and an imaging profile consistent with meningioma.

There have been prior reports of LCHs originating in the dura $^{3)}$ and the tentorium ${ }^{5)}$ of the cerebellum ${ }^{5)}$. Cranial and intracranial changes associated with LCH include : 1) lesions of the craniofacial bone and skull base, with or without soft-tissue extension; 2) intracranial and extra-axial changes in the hypothalamic-pituitary region, meninges, and other circumventricular organs, including the pineal gland, choroid plexus, and ependyma; 3 ) intra-axial parenchymal disease in the gray matter or white matter, with striking symmetric lesions and a predominant neurodegenerative pattern in the cerebellum and basal ganglia; and 4) localized or diffused cerebral atrophy ${ }^{9}$.

Meningiomas are relatively common tumors that occur most frequently on intracranial meninges. The World Health Organization (WHO) classification of central nervous system tumors defines various types of meningiomas. Most are slow-growing (WHO grade I), including some rare subtypes such as microcystic meningioma, secretory meningioma, and metaplastic meningioma. Brain-invasive (WHO grade II), atypical (WHO grade II), and anaplastic (WHO grade III) meningiomas are more aggressive $e^{7}$. Specifically, osseous destruction can occur in atypical malignant meningiomas or hyperostosis-associated benign meningiomas, and approximately $25 \%$ of meningiomas show psammomatous calcification. Typically, benign meningio- mas appear as round or elongated extra-axial masses with a broad dural attachment and have a density similar to that of the cerebrum in CT images. Enhanced scans of meningiomas show a dural tail sign, which indicates the presence of neoplastic dural infiltration and/or reactive vascularization of the adjacent dura. Low-intensity signals produced by calcification or vascular flow voids may be observed within the tumor ${ }^{10)}$. In addition to the typical meningioma signs, the $\mathrm{LCH}$ mass in the present case also presented with a slight defect of the adjacent intracranial skull consistent with atypical meningioma.

\section{CONCLUSION}

This report presents a case of dura-originating calvarial LCH that mimicked the imaging characteristics of a meningioma, including the typical dural tail sign. This case underscores the importance of a thorough histological biopsy and immunocytochemical examination in head tumor differential diagnosis.

\section{References}

1. Anagnostou E, Papageorgiou SG, Potagas C, Alexakis T, Kalfakis N, Anastasopoulos D : Square-wave jerks and smooth pursuit impairment as subtle early signs of brain involvement in Langerhans' cell histiocytosis. Clin Neurol Neurosurg 110 : 286-290, 2008

2. Aricò $\mathrm{M}:$ Langerhans cell histiocytosis in adults : more questions than answers? Eur J Cancer 40 : 1467-1473, 2004

3. Baba T, Ibayashi Y, Morimoto S, Niwa J, Tanabe S, Hashi K : [A case of dural type of histiocytosis $\mathrm{X}$ presenting as a mass lesion in the tentorium cerebelli]. No Shinkei Geka 22 : 471-476, 1994

4. D’Ambrosio N, Soohoo S, Warshall C, Johnson A, Karimi S : Craniofacial and intracranial manifestations of langerhans cell histiocytosis : report of findings in 100 patients. AJR Am J Roentgenol 191 : 589-597, 2008

5. Holbrook TJ, Fogo A, Smith HP : Histiocytosis X involving the cervical dura. Childs Nerv Syst 3 : 50-52, 1987

6. Kasper EM, Aguirre-Padilla DH, Alter RY, Anderson M : Histiocytosis $\mathrm{X}$ : Characteristics, behavior, and treatments as illustrated in a case series. Surg Neurol Int $2: 57,2011$

7. Louis D, Ohgaki H, Wiestler OD, Cavenee WK, Burger PC, Jouvet A, et al. : The 2007 WHO Classification of Tumours of the Central Nervous System. Acta Neuropathol 114: 97-109, 2007

8. Mosiewicz A, Rola R, Jarosz B, Trojanowska A, Trojanowski T : Langerhans cell histiocytosis of the parietal bone with epidural and extracranial expansion - case report and a review of the literature. Neurol Neurochir Pol 44 : 196-203, 2010

9. Prayer D, Grois N, Prosch H, Gadner H, Barkovich AJ : MR imaging presentation of intracranial disease associated with Langerhans cell histiocytosis. AJNR Am J Neuroradiol 25 : 880-891, 2004

10. Saloner D, Uzelac A, Hetts S, Martin A, Dillon W : Modern meningioma imaging techniques. J Neurooncol 99 : 333-340, 2010 